\title{
Open-Label Clinical Trials of Oral Pulse Dexamethasone for Adults with Idiopathic Nephrotic Syndrome
}

\author{
Monique E. Cho ${ }^{a}$ Mary H. Branton ${ }^{a}$ David A. Smith ${ }^{a}$ Linda Bartlett ${ }^{a}$ \\ Lilian Howard $^{a}$ James C. Reynolds ${ }^{b}$ Donald Rosenstein ${ }^{c}$ Sanjeev Sethi ${ }^{d}$ \\ M. Berenice Nava ${ }^{a}$ e $\quad$ Laura Barisoni $^{f} \quad$ Fernando C. Fervenzag ${ }^{g}$ Jeffrey B. Kopp ${ }^{a}$ \\ ${ }^{a}$ Kidney Disease Section, National Institute of Diabetes and Digestive and Kidney Disease, Bethesda, MD, USA; \\ ${ }^{\mathrm{b}}$ Radiology and Imaging Services, Mark O. Hatfield Clinical Research Center, Bethesda, MD, USA; ${ }^{\mathrm{C}}$ Department of \\ Psychiatry, University of North Carolina, Chapel Hill, NC, USA; ${ }^{d}$ Department of Pathology, Mayo Clinic, Rochester,

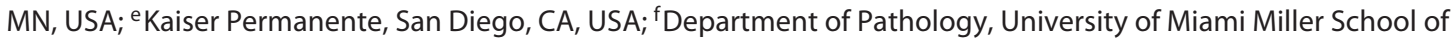 \\ Medicine, Miami, FL, USA; ${ }^{9}$ Division of Nephrology and Hypertension, Mayo Clinic, Rochester, MN, USA
}

\section{Keywords}

Steroids · Glucocorticoids · Proteinuria · Remission

\begin{abstract}
Background: In adults with primary focal segmental glomerulosclerosis (FSGS), daily prednisone may induce complete remissions (CR) and partial remissions (PR), but relapses are frequent and adverse events are common. Methods: We carried out 2 open-label, uncontrolled trials to explore the efficacy and tolerability of pulse oral dexamethasone as an alternative to daily prednisone. We enrolled adult patients with proteinuria $>3.5 \mathrm{~g} /$ day despite the use of renin-angiotensin-aldosterone blockade. In the first trial, we enrolled 14 subjects with FSGS and administered 4 dexamethasone doses $\left(25 \mathrm{mg} / \mathrm{m}^{2}\right)$ daily for 4 days, repeated every 28 days over 32 weeks. The second trial involved a more intensive regimen. Eight subjects received 4 dexamethasone doses of $50 \mathrm{mg} / \mathrm{m}^{2}$ every 4 weeks for 12 weeks, followed by 4 doses of $25 \mathrm{mg} / \mathrm{m}^{2}$ every 4 weeks for 36 weeks; subjects were randomized to 2 doses every 2 weeks or 4 doses every 4 weeks. Results: In the first trial, we enrolled 13 subjects with FSGS
\end{abstract}

\section{KARGER}

Published by S. Karger AG, Basel

E-Mail karger@karger.com

www.karger.com/ajn and 1 with minimal change disease and found a combined CR and PR rate of $36 \%$. In the second trial, we enrolled 8 subjects. The combined CR and PR rate was 29\%. Analysis combining both trials showed a combined CR and PR rate of 33\%. Adverse events were observed in $32 \%$ of subjects, with mood symptoms being most common. There were no serious adverse events related to the study. Conclusion: We conclude that high dose oral dexamethasone is well tolerated by adults with idiopathic nephrotic syndrome and may have some efficacy.

Published by S. Karger AG, Basel

\section{Introduction}

Focal segmental glomerulosclerosis (FSGS), including collapsing glomerulopathy, is the most common cause of primary nephrotic syndrome in adults. FSGS can be clas-

This is a work of the U.S. Government and is not subject to copyright protection in the United States. Foreign copyrights may apply. 
sified into 6 forms: primary, adaptive, APOL1-associated, high-penetrance genetic variant-associated, viral infection-associated, and medication-associated forms [1]. Conventional treatment for primary FSGS typically begins with daily prednisone or less commonly alternate day prednisone.

Among FSGS patients who receive glucocorticoids as the initial treatment, $61 \%$ enter complete remission (CR) following a prolonged initial treatment compared to $15 \%$ who received a short treatment of 16 weeks [2]. Such prolonged high-dose steroid course has substantial toxicity and significant morbidity. Nevertheless, a recent retrospective case series from the University of North Carolina suggested more favorable outcomes, with a hazard ratio for renal survival of 0.49 (95\% CI 0.28-0.86) in subjects with FSGS who received high-dose glucocorticoid therapy for a median of 3.0 months (interquartile range [IQR] 1.5-5.9 months) [3].

The mechanism by which glucocorticoid therapy induces remission has long remained unknown. There are at least 2 possibilities: these agents modulate immune functions and/or have a direct effect on podocytes. A recent report suggested that in FSGS and MC, glucocorticoid sensitivity is due to an expansion of the $\mathrm{Cd} 11 \mathrm{~b}^{+}$ HLA-DR ${ }^{-} \mathrm{CD} 14^{-} \mathrm{CD} 15^{+}$cell population; these cells are immature myeloid-derived stem cells that can suppress $\mathrm{T}$ cell responses [4]. This would add glucocorticoid-sensitive FSGS and MCD to the list of inflammatory and autoimmune diseases in which myeloid-derived stem cells are believed to play a role, including type 1 diabetes, multiple sclerosis, and inflammatory bowel disease [5].

Recent work suggests that dexamethasone has direct effects on cultured human podocytes, which express an intact glucocorticoid signaling pathway [6]. Xing et al. [7] showed that dexamethasone activates glucorticoid signaling in human podocytes. Working at both transcriptional and posttranscriptional levels [6], Xing et al. [7] demonstrated that dexamethasone increases the expression of nephrin and downregulates expression of vascular endothelial growth factor, the latter being known to be upregulated in minimal change disease.

Pulse oral dexamethasone has been used in several conditions. As initial therapy for acute and chronic immune thrombocytopenia, high-dose dexamethasone (40 mg/ day) administered to patients as a single dose daily for 4 consecutive days, every 14 days for 4 courses, has shown efficacy equivalent to daily prednisone [8]. As therapy for multiple myeloma, oral pulse dexamethasone was administered at $20 \mathrm{mg} / \mathrm{m}^{2} /$ day for 4 days of each 35-day cycle. Downward dose adjustment was required in $8 \%$ of patients because of adverse events. Complications severe enough to require hospitalization, including pancreatitis, perforated viscus, or pneumonia were seen in 4 of 112 subjects. Candidiasis, herpetic lesions, exacerbation of diabetes, or proximal muscle weakness each occurred in $<5 \%$ of patients [9]. Current therapy for multiple myeloma involves lower doses of dexamethasone in combination with a second medication. In a study involving patients with chronic inflammatory demyelinating polyradiculoneuropathy, pulse oral dexamethasone was found to have fewer adverse events compared to standard prednisolone treatment [10]. These studies led us to examine whether dexamethasone, used for FSGS, might have similar efficacy and reduce toxicity compared to the standard approach to daily prednisone use.

In this report, we present the results of 2 trials using pulse oral dexamethasone administered over 32 weeks (study 1) and 48 weeks (study 2) as initial immunosuppressive therapy for adult patients with primary FSGS. These trials were open-label, uncontrolled studies designed to develop preliminary evidence of efficacy and safety that would justify future controlled studies of this approach.

\section{Methods}

Human Subjects Review

The protocols for study 1 and 2 were approved in advance by the appropriate institutional review boards at the National Institute of Diabetes and Digestive and Kidney Diseases, NIH, and at Mayo Clinic. All subjects gave written informed consent. The studies were listed at the US Federal Clinical Trials.gov website as NCT00004990.

\section{Entry Criteria}

We enrolled patients with primary FSGS (including the variants tip lesion, collapsing variant, and not otherwise specified variant) and MCD. Adaptive FSGS was excluded based on the presence of characteristic features, including normal or near normal serum albumin levels despite nephrotic-range proteinuria, enlarged glomeruli, perihilar sclerosis and hyalinosis, and limited podocyte foot process effacement. Patients with possible medication-associated FSGS, such as those who had received lithium or interferon alpha, were excluded.

Entry criteria also included nephrotic range proteinuria, defined as urine protein $\geq 3.5 \mathrm{~g} / 1.73 \mathrm{~m}^{2} /$ day, and Chronic Kidney Disease Epidemiology Collaboration (CKD-EPI) glomerular filtration rate $(\mathrm{GFR}) \geq 40 \mathrm{~mL} / \mathrm{min} / 1.73 \mathrm{~m}^{2}$ at the time of entry while receiving maximally tolerated doses of angiotensin converting enzyme (ACE) inhibitor or angiotensin receptor blocker (ARB) for at least 4 weeks prior to study entry and prior steroid use was $<8$ weeks of daily or alternate day steroids at $>0.5 \mathrm{mg} / \mathrm{kg}$. Exclusion criteria included patients with poorly controlled diabetes mellitus, poorly controlled hypertension ( $>25 \%$ of values $>125 / 75$ $\mathrm{mm} \mathrm{Hg}$ ), HIV-1 infection, hepatitis C infection, hepatitis B infection, and untreated Mycobacterial infection. Pregnant or nursing 
Table 1. Study treatment protocols

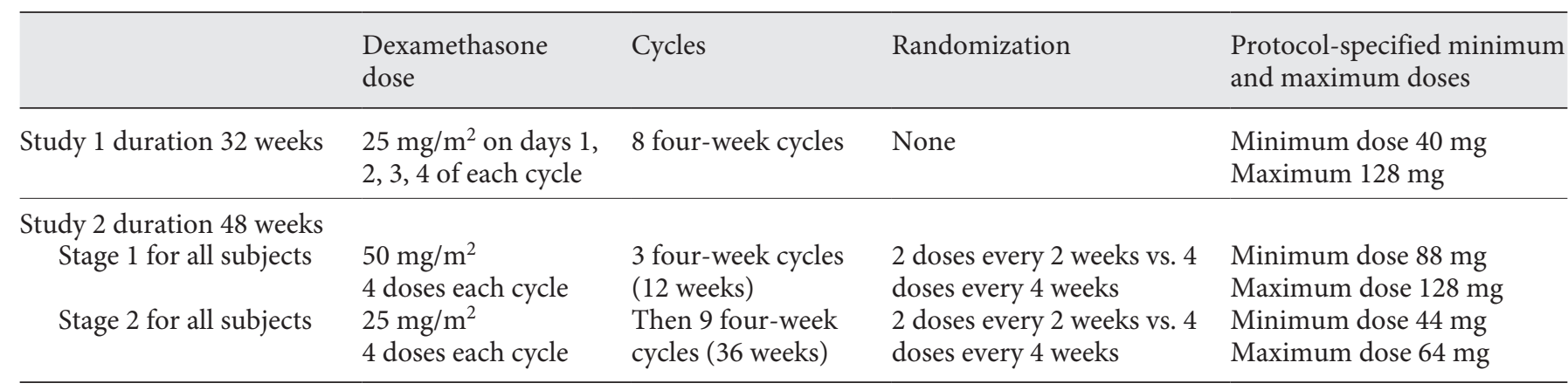

In study 1 , subjects received eight 4 -week cycles of dexamethasone. In study 2 , subjects were received a stage 1 (high) dose for 12 weeks and the stage 2 (lower) dose for 36 weeks. The lowest and highest doses administered are shown.

women and those unwilling or unable to use effective contraception were excluded.

\section{Kidney Biopsies}

After study conclusion, kidney biopsies performed prior to study enrollment from 21 subjects were reviewed and classified according to histologic variant. The slide sets were reviewed by either of 2 nephropathologists. Two slide sets had been destroyed, and 1 slide set was unavailable for review; the original biopsy interpretations were collapsing glomerulopathy; FSGS, NOS; and MCD; and these interpretations were retained. Diagnostic categories of FSGS and collapsing glomerulopathy were applied following the Columbia FSGS classification [11].

\section{Intervention}

In study 1 (32-week duration), subjects were given oral dexamethasone $25 \mathrm{mg} / \mathrm{m}^{2} /$ day, on days 1-4 of each 28-day cycle, for a total of 8 cycles. The minimum daily dose was established as $40 \mathrm{mg}$ and the maximum dose as $128 \mathrm{mg}$. The doses were selected as being similar to doses used for thrombocytopenia and multiple myeloma described above. The study drug supplies were maintained at the NIH Clinical Center pharmacy, and patients received a supply of dexamethasone for the next treatment cycle at each monthly visit.

Blood pressure medications were adjusted to maintain baseline blood pressure. ACE inhibitor, ARB, or non-dihydropyridine calcium channel blockers were not started during the study, as these medications modify proteinuria. All patients were given $1,500 \mathrm{mg}$ elemental calcium and 800 units of vitamin D daily while taking dexamethasone to minimize glucocorticoid-related bone loss.

In order to examine whether length and dose of treatment had an effect on proteinuria, the dose and duration of dexamethasone were increased in study 2 (48-week duration). Subjects received doses of $50 \mathrm{mg} / \mathrm{m}^{2}$ for 12 weeks (minimum dose $88 \mathrm{mg} /$ day and maximum dose $128 \mathrm{mg} /$ day) and then doses of $25 \mathrm{mg} / \mathrm{m}^{2}$ for the remaining 36 weeks (minimum dose $44 \mathrm{mg}$ and maximum dose $64 \mathrm{mg}$ ). Subjects were randomized (1:1) using a stratified block design to 2 doses every 2 weeks versus 4 doses every 4 weeks for both periods. The increase in doses and duration from the first to the second study was based on estimate of how much the dexamethasone dose could be increased while preserving a tolerable safety profile. Treatment regimens are summarized in Table 1.

Dexamethasone for Nephrotic Syndrome

\section{Efficacy Outcomes}

The primary outcome was prespecified as the combination CR and partial remission rate (PR) at end of study (EOS). Baseline proteinuria was determined as the mean value obtained from 3 urine collections collected within 8 weeks of commencing glucocorticoid therapy. In addition, long-term remission status was evaluated 24 months after EOS and again after a mean of 5.4 years after study initiation.

$\mathrm{CR}$ was defined as proteinuria $<0.3 \mathrm{~g} /$ day. $\mathrm{PR}$ was defined as a $50 \%$ fall in proteinuria compared to baseline, proteinuria $<3.5 \mathrm{~g} /$ day, and a preserved estimated GFR (eGFR), specified as $>60 \%$ of baseline value. Importantly, this definition of PR has been shown to correlate with improved long-term renal survival in adults with FSGS, compared to those who fail to experience a PR [12]. Limited response (LR) was defined as a $50 \%$ fall in proteinuria compared to baseline proteinuria; our rationale for defining this exploratory outcome was that we believed it was worth noting substantial reductions in proteinuria that did not meet full criteria for a PR. Preserved eGFR during treatment was defined as an eGFR of at least a $75 \%$ of baseline value, it was calculated using the CKD-EPI calculation. Preserved eGFR at last evaluation was defined as eGFR of at least a $60 \%$ of baseline value using the CKD-EPI calculation. The definitions of LR and preserved eGFR for long-term follow-up have not been validated and were intended for exploratory purposes only. All other outcomes were described as nonresponse (NR).

Only urine collections that had an adequate creatinine excretion were used to determine baseline proteinuria and proteinuria outcomes; adequate creatinine excretion was defined as a urine collection that had between 75 and $125 \%$ of the average creatinine excretion of all urine collections for that subject, evaluated retrospectively. During follow-up, proteinuria was assessed using either a $24 \mathrm{~h}$ urine collection or a urine protein/creatinine ratio, multiplied by the average creatinine excretion in that subject to generate an estimated daily protein excretion.

\section{Safety Outcomes}

Adverse events associated with glucocorticoid therapy were evaluated in the following 5 domains. Following the definition of the US Food and Drug Administration, serious adverse events were defined as those that involve death are life threatening, re- 
quire hospitalization, result in disability or permanent damage, involve a congenital anomaly, or require intervention to prevent permanent damage or impairment.

\section{Adrenal Suppression}

An adrenocorticotrophic hormone (ACTH) stimulation test at baseline, follow-up visits, and EOS were performed. Serum cortisol levels were determined 30 and $60 \mathrm{~min}$ after administration of ACTH $0.25 \mu \mathrm{g}$ intravenously. A plasma cortisol of $>18 \mu \mathrm{g} / \mathrm{dL}$ and an increase from baseline of $>7 \mu \mathrm{g} / \mathrm{dL}$ at either 30 or $60 \mathrm{~min}$, respectively, were considered a normal response.

\section{Cushingoid Appearance}

Photographs were taken of the patient's face and body from both the front and the side. These pictures were taken in their underwear, shorts, and tank tops. The photographs were assessed by the investigators masked as to the status of the photograph (baseline vs. EOS) for steroid treatment changes in fat distribution.

\section{Ophthalmologic Complications}

Subjects had a slit lamp evaluation done by an ophthalmologist for the presence of cataracts and elevated intraocular pressure at baseline, 10 weeks, 22 weeks, and at EOS.

General Health and Psychological Survey Instruments

Four instruments, SF-36, Young Mania Rating Scale (YMRS), Delirium Rating Scale-R-98 (DRS-R-98), and beck depression inventory-II (BDI-II) were used to assess general health (quality of life) and psychiatric symptoms at baseline and EOS. Baseline evaluation included a psychiatric history to elicit past or present symptoms suggestive of depression or psychosis. All questionnaires were administered to all subjects by research personnel, with the exception of the BDI-II, which was self-administered and completed at either NIH or at Mayo Clinic.

The SF-36 consists of an 8-scale profile of functional health and well-being; scores range from 0 to 100 , with 0 representing selfreported poorest-health and 100 representing best health. The YMRS was used to measure patient's mood state, and it consists of an 8-point global rating that includes eleven questions each with defined grades of severity [13]. A YMRS score of $0-1$ indicates euthymic; $2-3$ indicates hypomanic; $4-5$ indicates manic, and a score of 6-7 indicates severely manic. A BDI-II was used to assess for the presence and severity of depression; a score of $0-12$ suggests nondepressed, a score of 13-19 suggests dysphoria, and a score of 2063 suggests dysphoric depressed [9]. The DRS-R-98 was used to evaluate delirium symptom severity. The DRS-R-98 scores fall into 3 clusters: a score of $0-7$ suggests no delirium; a score of $8-13$ suggests subclinical/prodromal delirium, and score $\geq 14$ : suggests delirium [14].

\section{Bone Health}

Bone density was assessed by dual photon densitometry (DEXA) of lumbar spine using anterior-posterior view and femur (total hip view) at baseline, at mid-point (16 or 24 weeks), and at EOS. Osteopenia was defined as bone mass score on DEXA scan of the lumbar (L) 1-L4 spine (anterior-posterior view) reduced by $\geq 2$ SD units and osteoporosis was defined as bone mass T score on baseline DEXA scan at L1-L4 spine reduced by $\geq 2.5$ SD units. Magnetic resonance imaging of the hips was performed at baseline and at EOS to assess for avascular necrosis.

\section{Statistical Analysis}

Data are presented as median with IQR. Statistical analysis included paired $t$ test, and analysis of variance was performed using Prism software (GraphPad, San Diego, CA, USA).

\section{Results}

\section{Study Population}

Fourteen subjects with FSGS enrolled in and completed study 1; 7 subjects with FSGS and 1 with MCD were enrolled in and completed study 2 . In study 2, 4 subjects were randomized to every 2 -week dosing, and 4 subjects were randomized to every 4 -week dosing. One subject in study 2 voluntarily withdrew from the study without receiving study medication and is not included in this report. Summary baseline demographic and clinical characteristic are summarized in online supplementary Table 1 (for all online suppl. material, see www.karger.com/ doi/10.1159/000497064). Of 17 cases for which data were available, 13 had reduced serum albumin levels (online suppl. Table 3). Kidney biopsy FSGS histologic variant data are provided in online supplementary Table 4 . While children were eligible for participation, no children were enrolled; the ages range from 24 to 71 years. The median age of patients in study 1 (46 years) was numerically greater than in study 2 (34 years). For all 14 subjects taken together, the median (IQR) for proteinuria was 66 (54, 82 ). The median proteinuria levels at baseline were similar, $9.7(7.9,12.3) \mathrm{g} /$ day in study 1 and $9.6(8.1,12.2) \mathrm{g} /$ day in study 2. The eGFR levels were similar at baseline. Baseline and follow-up eGFR are presented as CKD-EPI eGFR, as this equation more accurately assesses GFR values over $60 \mathrm{~mL} / \mathrm{min} / 1.73 \mathrm{~m}^{2}$ [15]. There were no notable differences in race, ethnicity, and gender between the 2 groups.

\section{Proteinuria Outcomes}

The primary study outcome was remission (CR plus PR) at EOS. PR occurred in 5 of 14 (36\%) of the subjects in study 1 and 2 of 7 (29\%) of subjects with FSGS in study 2 ; the overall study remission rate was 33\% (online suppl. Table 2). The remaining subjects had LR or NR. When the subject with MCD is included (she has only an LR, somewhat surprisingly), the response rates for the subjects in study 2 were $25 \%$. In the pooled study group, proteinuria decreased from $9.5(7.7,12.3) \mathrm{g} /$ day at base to $4.3(3.4,7.6)$ g/day at EOS ( $p \leq 0.05$; Fig. 1 and online suppl. Fig. 2). There was no CR at EOS. The median eGFR at EOS was unchanged from baseline $\left(66 \mathrm{~mL} / \mathrm{min} / 1.73 \mathrm{~m}^{2}\right)$. 
Fig. 1. Proteinuria at baseline, EOS, and last evaluation. Shown are the mean 24 -h protein excretion rates at baseline, at EOS and at last evaluation. Baseline and EOS outcomes for 21 subjects, 1 subject was withdrawn from study. At last follow-up evaluation, 1 subject was lost to follow-up and 1 died from pancreatitis 8 years after completion of study. EOS, end of study.

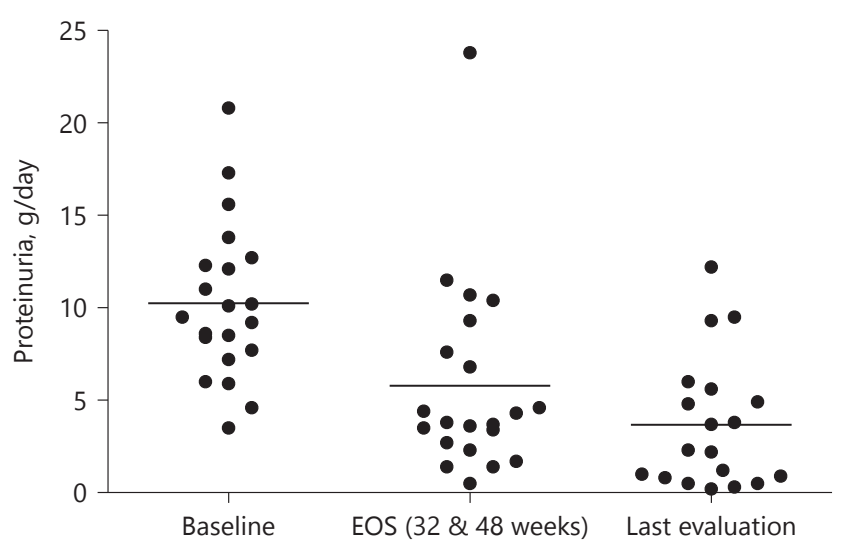

tion. Subject 7 died of pancreatic cancer; the other subjects were alive at last evaluation. No subject progressed to ESKD during treatment period or follow-up.

\section{Adverse Events}

Adverse events are summarized in Table 2. The most common side effects were mood symptoms followed by sleep disturbances (particularly insomnia). Mood symptoms occurred in 3 of 14 (21\%) subjects in study 1 and 3 of 8 (38\%) subjects in study 2 . Mood symptoms included the following: irritability, aggressive behavior, short temper, and there was one episode of road rage that was found not to be related to the study. One subject had an episode of a mild increase intraocular pressure that was treated with brimonidine eye drops. The episode of road rage, herpes simplex recurrent infection, and increased intraocular pressure, all occurred to the same subject in study 2 . The subject remained in the study after evaluation by dermatology, ophthalmology, and psychiatry. Herpes simplex was the only infection that occurred during the study. Acne was not observed.

There were 2 serious adverse events, both of which were judged to be unrelated to study medication: acute onset of appendicitis requiring appendectomy and a biceps tendon rupture. The appendicitis occurred 3 months after the start of the study. The tendon rupture occurred in an avid exercise enthusiast 3 months after EOS. One subject developed increased fasting glucose, Cushingoid appearance, and weight gain and was withdrawn after 24 weeks of treatment on the every- 4 week arm in study 2 ; the subject had not developed overt diabetes at 5 year follow-up. All subjects had a normal adrenal response to ACTH stimulation test at baseline and EOS. No subject developed cataract during the study. Overall perceptions of health were evaluated with an 
Table 2. Adverse events

\begin{tabular}{|c|c|c|c|c|}
\hline Number & 14 & 4 & 4 & 22 \\
\hline Headache & $1(7)$ & $1(25)$ & 0 & $2(9)$ \\
\hline Sleep disturbances & $3(21)$ & $1(25)$ & $1(25)$ & $5(23)$ \\
\hline Mood swings & $3(21)$ & $2(50)$ & $1(25)$ & $6(27)$ \\
\hline Impaired glucose tolerance & $1(7)$ & 0 & $1(25)$ & $2(9)$ \\
\hline Cushingoid appearance & $1(7)$ & 0 & $1(25)$ & $2(9)$ \\
\hline Muscle pains & $1(7)$ & $1(25)$ & 0 & $2(9)$ \\
\hline Infections & 0 & $\begin{array}{l}1(25) \\
\text { Herpes Zoster }\end{array}$ & 0 & 0 \\
\hline Serious adverse event & 0 & $\begin{array}{l}1(25) \\
\text { Tendon rupture }\end{array}$ & $\begin{array}{l}2(50) \\
\text { Acute appendicitis increased } \\
\text { intraocular pressure }\end{array}$ & $3(14)$ \\
\hline
\end{tabular}

The number and percentage of subjects with common adverse events are shown.

Mood swings included irritability, anxiety, aggressive behavior, short temper and one episode of road rage. No one develop overt diabetes.

SF-36 survey, completed at baseline and EOS, in which higher scores denotehigher function and the maximal score in each domain is 100 (Fig. 2; online suppl. Table 5). Data are limited to 19 subjects who completed 2 questionnaires; 3 subjects are not represented, including the subject who withdrew. The only domains that reached or approached statistical significance at the EOS were improvements in mental health $(p<0.01)$ and vitality/energy $(p<0.06)$.

\section{Psychological Function}

Mental health was specifically evaluated with 3 survey instruments (Fig. 2). On the BDI-II, mean score was $5.76 \pm 5.5$ vs. $2.43 \pm 2.6$ at baseline and EOS, respectively $(p<0.05)$. Scores from 0 to 9 represent no depressive symptoms; 6 subjects exceeded these values $(10,12,13$, and 15, both obtained at baseline). The mean YMRS score was $1.0 \pm 1.6$ at baseline, increased to $1.95 \pm 2.3$ at 4 weeks and decreased to $0.6 \pm 1.1$ at EOS ( $p$ values by analysis of variance, with comparison of baseline to 4 weeks and baseline to EOS were $p=0.10, p=0.96$, respectively). The DRS-R-98 showed similar results at baseline and EOS, all subjects having no signs or symptoms of delirium.

\section{Bone Health}

Bone mass was assessed by DEXA scan at hip and spine at 3 points: baseline, study mid-point ( 16 weeks for study 1 and 24 weeks for study 2), and EOS. Three individuals had missing baseline DEXA scans, and 6 individuals had missing EOS DEXA scans; therefore, we limited our analysis to 13 subjects with both baseline and EOS scans. No subject had osteopenia at any time point, and the changes from baseline to either mid-point or EOS were not significant (online suppl. Fig. 2). One subject in study 2 had a bone mass reduction of $1.3 \mathrm{SD}$ units at L1-L4 site at EOS. All subjects underwent hip magnetic resonance imaging at the start and at the end of the study; no subjects developed avascular necrosis. One subject exceeded the weight limit for the scan; all others were studied at EOS.

\section{Discussion}

Idiopathic nephrotic syndrome, and primary FSGS in particular, represents a challenging entity for nephrologists, with most therapies having limited success and substantial toxicity. Initial treatment for adults involves daily prednisone administered for 2-4 months, at a dose of $1 \mathrm{mg} / \mathrm{kg}$ per day [16]. In the present report, we have evaluated an alternative regimen using pulse oral dexamethasone in a study group comprised of 21 FSGS subjects and $1 \mathrm{MCD}$ subject. Our results suggest that intermittent oral dexamethasone induces $\mathrm{PR}$ rate of $33 \%$ at EOS. Induction of CR plus PR following dexamethasone therapy was not predictive of long-term follow-up outcomes. Therapy 


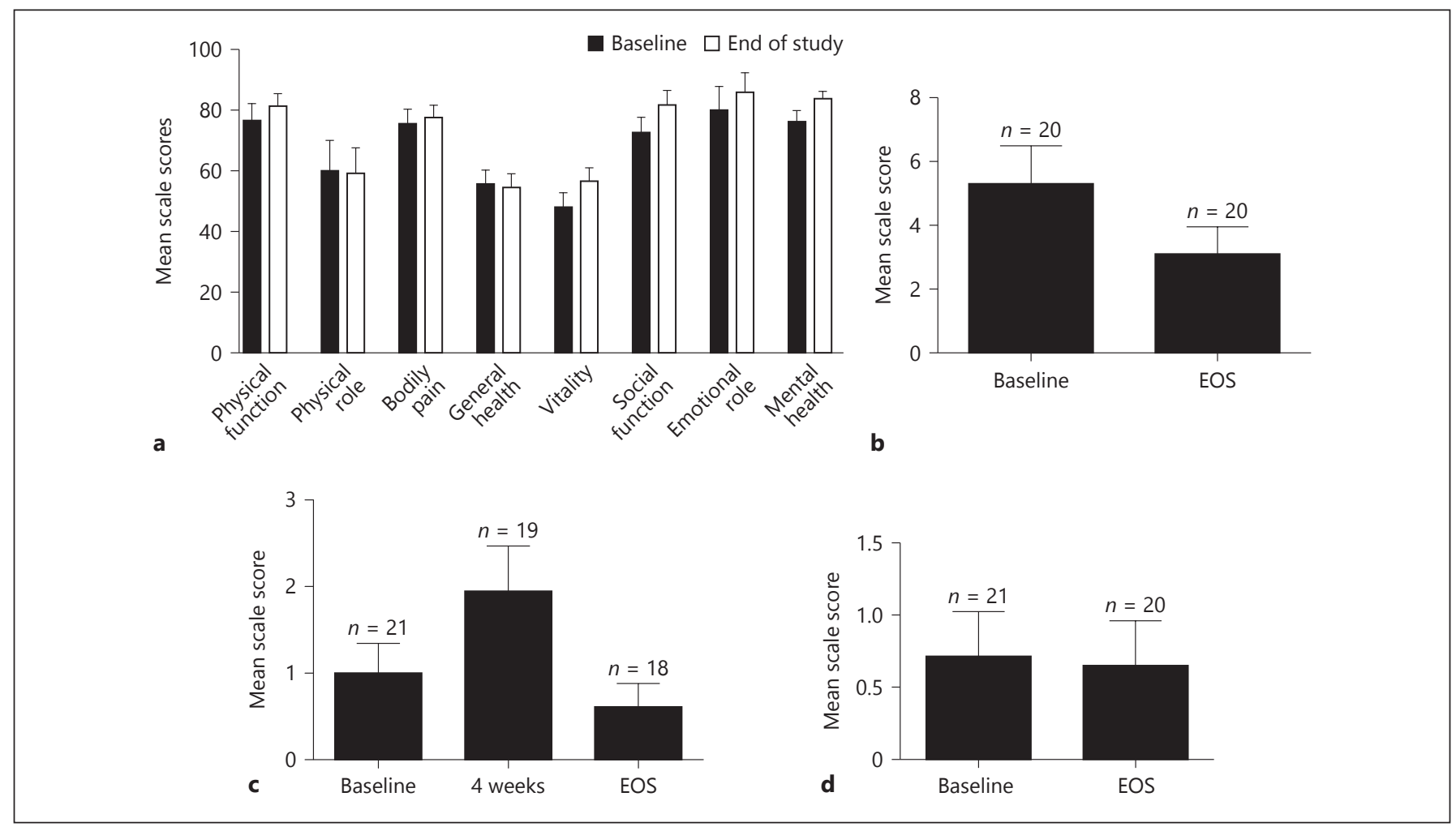

Fig. 2. Results of survey instruments for general health and psychological health. Presented are the scores, as mean \pm SD. a SF-36, b BDI-II, c YMRS, and $\mathbf{d}$ DRS at baseline, 4 weeks (for the mania scale) and EOS. BDI-II, beck depression inventory II; YMRS, young mania rating scale; DRS, delirium rating scale; EOS, end of study; SF-36, Short-form health survey. was generally well-tolerated. Our study has several strengths. First, this is the first study to explore oral pulse glucocorticoids for primary FSGS. Second, we determined short-term outcomes (up to 24 months after completing therapy) in nearly all subjects and as well as longterm follow-up outcomes (median of 5.4 years) in most subjects. Third, we explored several potential adverse events, involving a range of testing.

Our study has several important limitations. First, this was an uncontrolled study, as have been most published studies using prednisone. Second, the sample size was small and was divided into 3 different treatment regimens; it is not possible to conclude whether any single regimen had an advantage over any other. Third, 3 patients were lost to follow-up at long-term evaluation.

The efficacy results demonstrated in the trials reported here should be placed in the context of the previouslypublished trials. Achieving a partial reduction in proteinuria and its maintenance has evidence of improving renal survival and slowing the rate of renal function decline [12]. One retrospective study suggested that FSGS patients with nephrotic range proteinuria may benefit from a longer length course of therapy with steroids [17]. Heavier proteinuria, particularly $>10 \mathrm{~g} /$ day, is associated with a higher risk of progression. In the present study, 10 (48\%) subjects presented with proteinuria in excess of 10 g/day. Four subjects attained PR rates at EOS and 3 sustained PR rates at 24 months follow-up after EOS; 1 of 4 reached CR with no additional immunosuppressive therapy; 2 of 4 remained PR at 7 years follow-up, and one progressed to end-stage kidney disease.

Korbet [18] suggested using 4 months of daily prednisone followed by a 4 months taper; this would provide an $80 \mathrm{~kg}$ adult with $13.5 \mathrm{~g}$ prednisone. Ponticelli et al. [2] suggested beginning with prednisone at $1 \mathrm{mg} / \mathrm{kg}$ for 16 weeks (assuming that there is some response after 8 weeks, to justify continuing therapy), followed by a slow taper (possibly over 20 weeks); in an $80 \mathrm{~kg}$ subject, this would total $9.4 \mathrm{~g}$ of prednisone. In a clinical study, they administered doses of $1 \mathrm{mg} / \mathrm{kg}$ for 8 weeks, followed taper, with a mean duration of 24 weeks, and reported a combined response rate of $68 \%$ (52\% CR, 16\% PR) [2]. Crook et al. [19] reported a series of African Americans with FSGS, treated with daily prednisone for a mean of 12 months, with cu- 
mulative prednisone dose of $10.8 \mathrm{~g}$. In this study, $60 \%$ achieved a urine $\mathrm{PCR}<2 \mathrm{~g} / \mathrm{g}$, suggesting $\mathrm{CR}$ or $\mathrm{PR}$. Of note, only $63 \%$ had entry uPCR of $>3 \mathrm{~g} /$ day, suggesting that many subjects were not nephrotic. Thus, 2 studies with daily prednisone have reported higher response rates compared to those in the present study. The decision to use daily prednisone versus pulse dexamethasone will require a balancing of the risks versus the benefits of each therapy.

How does the glucocorticoid dose employed here compare to comparable doses associated with conventional prednisone regimens recommended for FSGS in adults? The treatment protocol in study 1 equates to $1.6 \mathrm{~g}$ of dexamethasone in an $80 \mathrm{~kg}$ subject, which is equivalent in anti- inflammatory potency to $10.6 \mathrm{~g}$ of prednisone (1 $\mathrm{mg}$ of dexamethasone is comparable to $6.6 \mathrm{mg}$ of prednisone). For the same idealized subject in study 2, the treatment protocol involved administering $2.8 \mathrm{~g}$ of dexamethasone, which is equivalent to $18.4 \mathrm{~g}$ of prednisone. Thus, glucocorticoid doses administered in study 1 , when adjusted for relative anti-inflammatory potency, are comparable to those used in conventional nephrology practice, while the doses used in study 2 are substantially higher. Our study, while not powered to show a difference between study 1 and 2, shows no trend toward improved outcomes with the higher dose regimen. We conclude that the dose used in study 1 may be helpful in designing for further trials of pulse oral dexamethasone.

We defined a LR as an exploratory outcome to see whether a $50 \%$ fall in proteinuria without reaching a subnephrotic level proteinuria. Our rationale was to identify subjects with substantial reduction in proteinuria who do not achieve a PR. The follow-up duration was too short to determine whether this will prove to be a useful approach for future studies.

The mechanisms by which glucocorticoids induce remission in FSGS are unclear. Glucocorticoids were introduced in the therapy for the podocytopathies largely based on empiric evidence of success, particularly for MCD. The responses to this therapy have been taken as evidence of support for an immune role in pathogenesis, and there is some evidence for this [4]. Also, dexamethasone has direct effects to podocyte phenotype in vitro, including increasing nephrin mRNA expression [20].

There are relatively few predictors of a favorable response to glucocorticoid therapy. One study reported the degree of tubulointerstitial changes and mean glomerular diameter were 2 significant predictors found to correlate with poor response to glucocorticoid therapy thus poor renal outcomes [21]. Alexopoulos et al. [22] studied the factors influencing the course and the response to treat- ment in 33 adults with primary FSGS, of whom $28 \%$ had a CR, 54\% PR, and 18\% did not respond, showed that only age and plasma creatinine at biopsy had an independent predictive value for renal survival in nephrotic patients and only the severity of interstitial fibrosis predicted response to treatment.

In general, the regimens we report here were well tolerated, with relatively few adverse events and mood symptoms being the most common complaint. Seven of the 22 subjects had at least one adverse event, 2 of which were considered serious but not related to therapy. Our results demonstrate that high dose of dexamethasone is well tolerated in adults with FSGS. Prednisone is the most widely accepted glucocorticoid used as initial therapy for adult FSGS, with reported $41 \%$ of subjects reporting serious adverse events: diabetes mellitus, hypertension exacerbation, cellulitis, and severe myopathy [23]. High-dose dexamethasone has been used as therapy for multiple myeloma, both accompanied with and without additional chemotherapeutic agents. The most common side effects are Cushingoid features and/or insomnia. Other effects such as oral candidiasis, herpes lesions, aggravation of diabetes, peptic ulcer, hiccoughs, or quadriceps weakness have been noted [9].

In conclusion, these 2 open-label trials involving 22 subjects suggest that oral pulse may have modest efficacy in adults with idiopathic nephrotic syndrome (mostly primary FSGS) and is generally well-tolerated with an acceptable safety profile. The study size was too small to reach definitive conclusions about efficacy, but the preliminary data are encouraging in this regard. This study justifies further studies on the use of pulse oral dexamethasone for idiopathic nephrotic syndrome, which could offer a more tolerable approach to a prolonged course of daily glucocorticoid therapy. We propose that a randomized trial should compare daily or alternate day prednisone versus intermittent oral dexamethasone.

\section{Acknowledgments}

This work was supported by the National Institute of Diabetes and Digestive and Kidney Disease Intramural Research Program under ZO1-DK04312. We gratefully acknowledge the assistance of the NIH Clinical Center Pharmacy with drug assignment and the ophthalmology and radiology physicians who were involved in patient care, Dr. Meryl Waldman for critical review of the manuscript.

\section{Disclosure Statement}

All authors declare no conflicts of interest. 


\section{References}

1 Deegens JK, Steenbergen EJ, Wetzels JF. Review on diagnosis and treatment of focal segmental glomerulosclerosis. Neth J Med. 2008 Jan;66(1):3-12.

2 Ponticelli C, Villa M, Banfi G, Cesana B, Pozzi C, Pani A, et al. Can prolonged treatment improve the prognosis in adults with focal segmental glomerulosclerosis? Am J Kidney Dis. 1999 Oct;34(4):618-25.

3 Laurin LP, Gasim AM, Poulton CJ, Hogan SL, Jennette JC, Falk RJ, et al. Treatment with Glucocorticoids or Calcineurin Inhibitors in Primary FSGS. Clin J Am Soc Nephrol. 2016 Mar;11(3):386-94.

4 Li L, Zhang T, Diao W, Jin F, Shi L, Meng J, et al. Role of Myeloid-Derived Suppressor Cells in Glucocorticoid-Mediated Amelioration of FSGS. J Am Soc Nephrol. 2015 Sep;26(9): 2183-97.

5 Boros P, Ochando J, Zeher M. Myeloid derived suppressor cells and autoimmunity. Hum Immunol. 2016 Aug;77(8):631-6.

6 Guess A, Agrawal S, Wei CC, Ransom RF, Benndorf R, Smoyer WE. Dose- and time-dependent glucocorticoid receptor signaling in podocytes. Am J Physiol Renal Physiol. 2010 Oct;299(4):F845-53.

7 Xing CY, Saleem MA, Coward RJ, Ni L, Witherden IR, Mathieson PW. Direct effects of dexamethasone on human podocytes. Kidney Int. 2006 Sep;70(6):1038-45.

8 Andersen JC. Response of resistant idiopathic thrombocytopenic purpura to pulsed highdose dexamethasone therapy. N Engl J Med. 1994 Jun;330(22):1560-4.

9 Alexanian R, Dimopoulos MA, Delasalle K, Barlogie B. Primary dexamethasone treatment of multiple myeloma. Blood. 1992 Aug; 80(4):887-90.
10 van Schaik IN, Eftimov F, van Doorn PA, Brusse E, van den Berg LH, van der Pol WL, et al. Pulsed high-dose dexamethasone versus standard prednisolone treatment for chronic inflammatory demyelinating polyradiculoneuropathy (PREDICT study): a doubleblind, randomised, controlled trial. Lancet Neurol. 2010 Mar;9(3):245-53.

11 D’Agati VD, Fogo AB, Bruijn JA, Jennette JC. Pathologic classification of focal segmental glomerulosclerosis: a working proposal. Am J Kidney Dis. 2004 Feb;43(2):368-82.

12 Troyanov S, Wall CA, Miller JA, Scholey JW, Cattran DC; Toronto Glomerulonephritis Registry Group. Focal and segmental glomerulosclerosis: definition and relevance of a partial remission. J Am Soc Nephrol. 2005 Apr; 16(4):1061-8

13 Young RC, Biggs JT, Ziegler VE, Meyer DA. A rating scale for mania: reliability, validity and sensitivity. Br J Psychiatry. 1978 Nov; 133(5):429-35.

14 Franco JG, Trzepacz PT, Mejía MA, Ochoa $\mathrm{SB}$. Factor analysis of the Colombian translation of the Delirium Rating Scale (DRS), Revised-98. Psychosomatics. 2009 May-Jun; 50(3):255-62.

15 Stevens LA, Schmid CH, Greene T, Zhang YL, Beck GJ, Froissart M, et al. Comparative performance of the CKD Epidemiology Collaboration (CKD-EPI) and the Modification of Diet in Renal Disease (MDRD) Study equations for estimating GFR levels above $60 \mathrm{~mL} /$ min/1.73 m2. Am J Kidney Dis. 2010 Sep; 56(3):486-95.
16 Korbet SM. Angiotensin antagonists and steroids in the treatment of focal segmental glomerulosclerosis. Semin Nephrol. 2003 Mar; 23(2):219-28.

17 Rydel JJ, Korbet SM, Borok RZ, Schwartz MM. Focal segmental glomerular sclerosis in adults: presentation, course, and response to treatment.AmJ Kidney Dis. 1995 Apr;25(4):534-42.

18 Korbet SM. Treatment of primary FSGS in adults. J Am Soc Nephrol. 2012 Nov;23(11): 1769-76.

19 Crook ED, Habeeb D, Gowdy O, Nimmagadda S, Salem M. Effects of steroids in focal segmental glomerulosclerosis in a predominantly African-American population. Am J Med Sci. 2005 Jul;330(1):19-24.

20 Takano Y, Yamauchi K, Hiramatsu N, Kasai A, Hayakawa K, Yokouchi M, et al. Recovery and maintenance of nephrin expression in cultured podocytes and identification of HGF as a repressor of nephrin. Am J Physiol Renal Physiol. 2007 May;292(5):F1573-82.

21 Shiiki H, Nishino T, Uyama H, Kimura T, Nishimoto K, Iwano M, et al. Clinical and morphological predictors of renal outcome in adult patients with focal and segmental glomerulosclerosis (FSGS). Clin Nephrol. 1996 Dec;46(6):362-8.

22 Alexopoulos E, Stangou M, Papagianni A, Pantzaki A, Papadimitriou M. Factors influencing the course and the response to treatment in primary focal segmental glomerulosclerosis. Nephrol Dial Transplant. 2000 Sep; 15(9):1348-56

23 Uzu T, Harada T, Sakaguchi M, Kanasaki M, Isshiki K, Araki S, et al. Glucocorticoid-induced diabetes mellitus: prevalence and risk factors in primary renal diseases. Nephron Clin Pract. 2007;105(2):c54-7. 